\title{
New case of secondary bladder amyloidosis with massive hematuria-role of intravesical instillations with dimethyl sulfoxide
}

\author{
Esther García Rojo ${ }^{1}$, Daniel A. González Padilla ${ }^{1}$, Alicia Castelo Loureiro ${ }^{2}$, Lucía Parrilla Rubio ${ }^{3}$, \\ Ángel Tejido Sánchez ${ }^{1}$, Félix Guerrero Ramos ${ }^{1}$ \\ ${ }^{1}$ Urology Department, ${ }^{2}$ Oncology Department, ${ }^{3}$ Pathology Department, University Hospital 12 de Octubre, Madrid, Spain \\ Correspondence to: Esther García Rojo. Department of Urology, University Hospital 12 de Octubre, Av. Córdoba SN, 29041, Madrid, Spain. \\ Email: esthergrojo@hotmail.com.
}

\begin{abstract}
Secondary bladder amyloidosis is a rare condition with less than 60 cases published in the world. It is usually secondary to chronic inflammatory processes such as rheumatologic diseases. Hematuria is its predominant and most important symptom, and usually occurs after a bladder catheterization. The diagnostic confirmation is made through a pathological and immunohistochemical study. The treatment must be staggered from less to more invasive. Our objectives are to present a new case of secondary bladder amyloidosis in a woman with a history of chronic bronchiectasis after tuberculosis and frequent super infections, whose main manifestation was a massive hematuria, and review this rare pathology. We have obtained very good initial results using intravesical instillations with dimethyl sulfoxide (DMSO) with complete resolution of the hematuria, the patient remaining asymptomatic for 6 months. After that, there was a recurrence of the hematuria that was treated with embolization of the hypogastric arteries, with good results. We can conclude that, despite being a rare condition, we must consider secondary bladder amyloidosis in patients who have already been diagnosed with systemic amyloidosis and/or chronic pathologies who develop hematuria after bladder catheterization. Based on our experience, instillations with dimethyl sulfoxide are a safe option and provide a quick and temporary resolution of hematuria symptoms.
\end{abstract}

Keywords: Secondary bladder amyloidosis; massive hematuria; dimethyl sulfoxide instillations

Submitted Jun 07, 2019. Accepted for publication Sep 10, 2019.

doi: 10.21037/tau.2019.09.22

View this article at: http://dx.doi.org/10.21037/tau.2019.09.22

\section{Introduction}

Amyloidosis (named for its staining similarities with starch) is an entity described by Rokitansky, but it owes its name to Wirchow, who named it this way in the midnineteenth century (1). It is a pathology characterized by an extracellular deposit of proteins called amyloidal material that with its progressive deposit, ends up causing an organ or tissue deterioration.

There are two types of amyloidosis based on location, localized and systemic. Furthermore, depending on the type of accumulated fibers, the disease can be classified into AL or primary amyloidosis and AA or secondary amyloidosis. In primary amyloidosis there is no evidence of a pathology responsible for the condition (except multiple myeloma) and it usually includes cardiovascular, gastrointestinal, skin, thyroid and lung involvement. AA or secondary amyloidosis, is related to a chronic inflammatory disease, being rheumatoid arthritis the most frequent cause, although in older papers it was tuberculosis (2) and typically comes with renal, suprarenal glands, lymphatic nodes and spleen affectation.

Other subtypes of amyloidosis are senile amyloidosis, amyloidosis associated with hemodialysis and AF amyloidosis, which is associated with a specific entity called familiar amyloidal polyneuropathy,

Its location in the genitourinary system is variable and can occur anywhere: kidneys, pelvis, ureters, bladder, urethra or even penis (3). The kidney is classically the most affected organ, especially in secondary amyloidosis, 
Table 1 Relevant family and personal history

\author{
Family history \\ Colon cancer in her mother \\ Personal history \\ Tuberculosis passed and treated in 1962 \\ Residual apical cavity in right lung and bilateral bronchiectasis with multiple previous bacterial colonizations \\ Chronic dyspepsia with antral atrophic gastritis with intestinal metaplasia \\ Infection by Helicobacter pylori treated in 2018
}

although it can also be affected in primary amyloidosis (4). Renal amyloidosis is one of the most frequent causes of nephrotic syndrome (5).

Bladder amyloidosis is a rare entity, being primary bladder amyloidosis more common than secondary (which is a rarity with less than 60 cases published in the world) $(6,7)$. The most preferred site of amyloid deposit is the posterior bladder wall (68\%), followed by the trigone (26\%) (8). Macroscopic hematuria, with or without clots, is the predominant and frequently the unique clinical manifestation, although it can also manifest as irritative voiding symptoms such as dysuria or pollakiuria (9). Without adequate treatment, the evolution of these patients can be complicated (10) and even fatal (11).

Regarding its endoscopic appearance, it is common to confuse it with an urothelial bladder tumor, sometimes with an infiltrating appearance, especially in cases of primary bladder amyloidosis (12-15). It can also be similar to a glandular cystopathy or inflammatory entities such as cystitis, being more typical in secondary cases (8). This is the reason why a histopathological study of the tissues is mandatory to obtain an accurate diagnosis.

The gold standard for the diagnosis is the histological and immunohistochemical study of the affected tissue, using special staining techniques with haematoxylin and/ or Congo Red to demonstrate the presence of amyloid material. The apple green birefringence is typical when observing the tissue in the polarizing microscope with the last-mentioned dye. It is possible to make a differentiation between the type of amyloidosis fibers using potassium permanganate $\left(\mathrm{KMnO}_{4}\right)$ : $\mathrm{AL}$ deposits tend to persist, while AAs are usually digested. Other techniques that can be used are immunohistochemical techniques or monoclonal antibodies (16).

Once the disease is confirmed, there is a wide range of therapeutic alternatives depending on the severity of the case: from observation and conservative management with continuous bladder irrigation with saline in mild cases, to more aggressive measures ranging from transurethral resections (TUR) with resection/cauterization of the affected areas, to embolization of the hypogastric arteries or, in severe cases, even to a partial or total cystectomy $(8,17)$.

Other measures that have been used for symptomatic control are intravesical instillations with different substances. One of them that seems to have had promising results is dimethyl sulfoxide (DMSO). DMSO has been shown in vivo to cause degradation of amyloid deposits $(18,19)$. Although its administration regimen is not clear $(6,20)$, it seems that together with a TUR of the lesions, it is the most effective treatment described in the literature in order to eliminate residual amyloid deposits and to achieve symptom control (6,21-23). Most of the treated cases were affected by primary bladder amyloidosis but it seems that this therapy is also effective on secondary amyloidosis deposits (22).

Regarding to subsequent follow-up, it is important to perform periodic cystoscopies to diagnose possible recurrences or possible coexisting malignancies $(20,24,25)$.

\section{Clinical case}

We present the case of a 71-year-old woman, born in Peru. Her family and personal history are summarized in Table 1 .

The patient arrives to the emergency room with epigastric abdominal pain that worsens with ingestion, accompanied by significant constipation and constitutional syndrome with loss of up to $10 \mathrm{~kg}$ of weight during a period of 6 months. No fever, vomiting, hematemesis, rectal bleeding or black stools.

Her relevant emergency blood tests results are, a creatinine of $1.46 \mathrm{mg} / \mathrm{dL}$, potassium of $3.72 \mathrm{mEq} / \mathrm{L}$, a microcytic anemia with $7.5 \mathrm{~g}$ of hemoglobin, a C-reactive 
Table 2 Diagnostic test and medical procedures

\begin{tabular}{ll}
\hline Studies & Findings \\
\hline Upper and lower Gl endoscopy & Chronic atrophic gastritis with areas suggestive of intestinal metaplasia. Biopsies are taken (Figure 1) \\
Thoraco-abdominal CT scan* & Bronchiectasis and a $5 \mathrm{~cm}$ bulla in the right upper lobe (Figure 2) \\
& Radionuclide content in the distal and blind ileum suggesting bleeding \\
& Urinary system without alterations (Figure 3) \\
& Regenerative anemia, without reticulocytosis. Normal B12, iron and folic acid \\
Anemia study & Positive Rouleax Coombs direct with high haptoglobin (excluding hemolysis) \\
& Bone marrow biopsy is performed \\
& Normal hormonal profile, study of malabsorption, celiac disease and autoimmunity \\
Complete blood test & HBV, HCV and HIV negative serologies \\
& Study of renal failure: proteinuria of $1.4 \mathrm{~g} / 24 \mathrm{~h}$ and albuminuria of 850.69 mg/g \\
& Culture with normal saprophyte flora and negative BAAR \\
\hline
\end{tabular}

*, CT is performed without intravenous contrast due to deterioration of renal function. Gl, gastrointestinal; CT, computed tomography; HBV, hepatitis B virus; HCV, hepatitis C virus; HIV, human immunodeficiency virus; BAAR, acid-alcohol resistant bacillus.
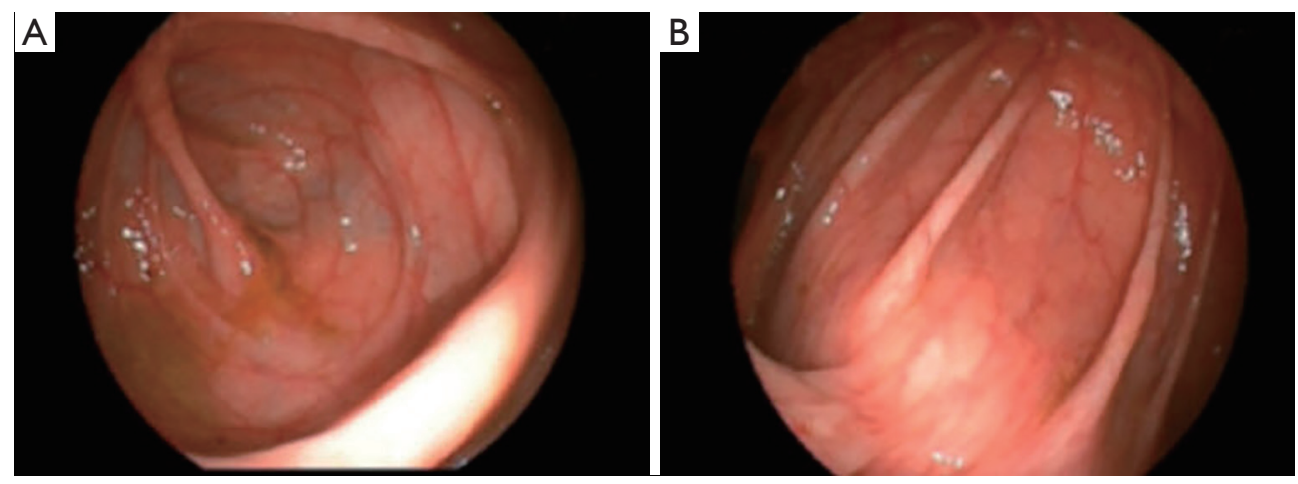

Figure 1 Colonoscopy images with mucosa of normal appearance.

protein of $19 \mathrm{mg} / \mathrm{dL}$ and a hypoalbuminemia of $2.19 \mathrm{~g} / \mathrm{L}$.

The subsequent study is summarized in Table 2.

During hospitalization, a bladder catheter is inserted for diuresis quantification, starting a macroscopic hematuria of significant amount, requiring a urological evaluation. The patient does not report previous episodes of hematuria. Conservative measures are taken, such as placement of a catheter for bladder washing with continuous irrigation of saline. In the following days the hematuria with significant anemia persists, requiring transfusion support and a cystoscopy, which is not useful due to the existence of a large organized clot occupying almost the entire bladder. Urgent exploration under anesthesia is decided, performing a cystoscopy with clots evacuation. We find a bladder with a lot of neovascularization, vascular tangles reminiscent of a chronic cystopathy and with multiple venous points that bleed during bladder distension. No exophytic lesions are observed. The bleeding points are coagulated and multiple randomized biopsies are taken (Figure 4).

After this intervention, the patient continues with severe hematuria, requiring transfusion of a total of 10 packed red blood cells. A new endoscopic surgery with coagulation of the bleeding vessels is performed.

Finally, we obtain the results of both gastrointestinal, bone marrow and bladder mucosa biopsies, all of them with AA amyloid deposits compatible with secondary amyloidosis (Figure 5).

Hematuria persists in the following days. After reviewing 

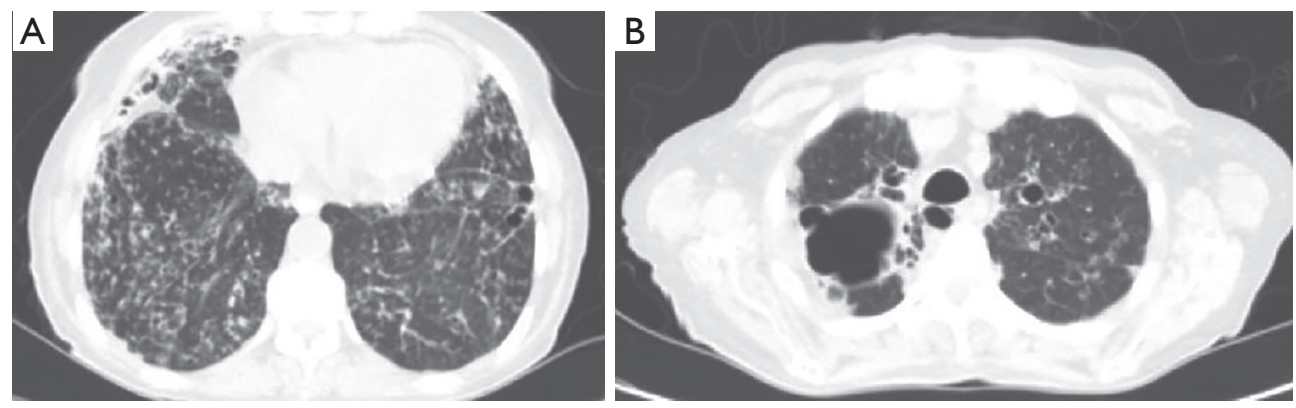

Figure 2 Thoracic CT images. (A) Bilateral pulmonary bronchiectasis; (B) bulla in right upper lobe.
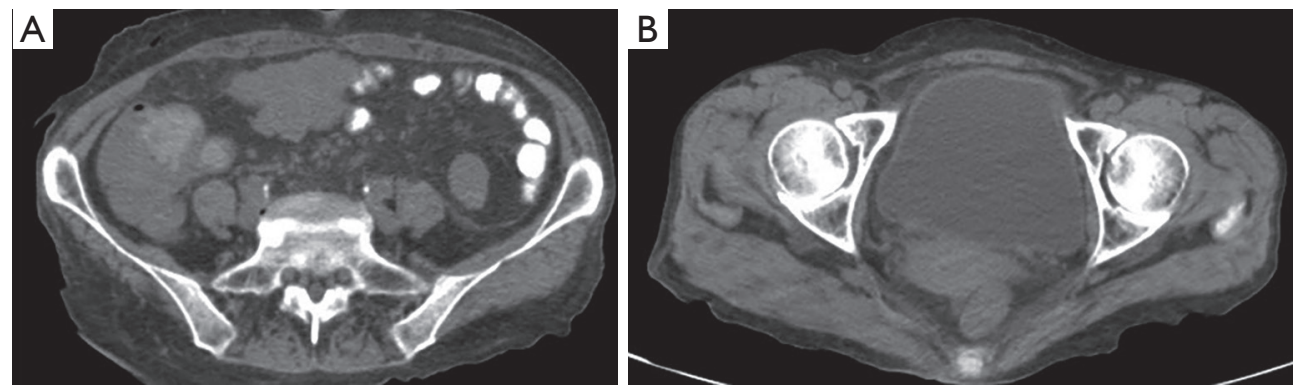

Figure 3 Abdominal-pelvic CT images. (A) Radiodense content in more distal portion of small intestine; (B) urinary bladder without alterations.
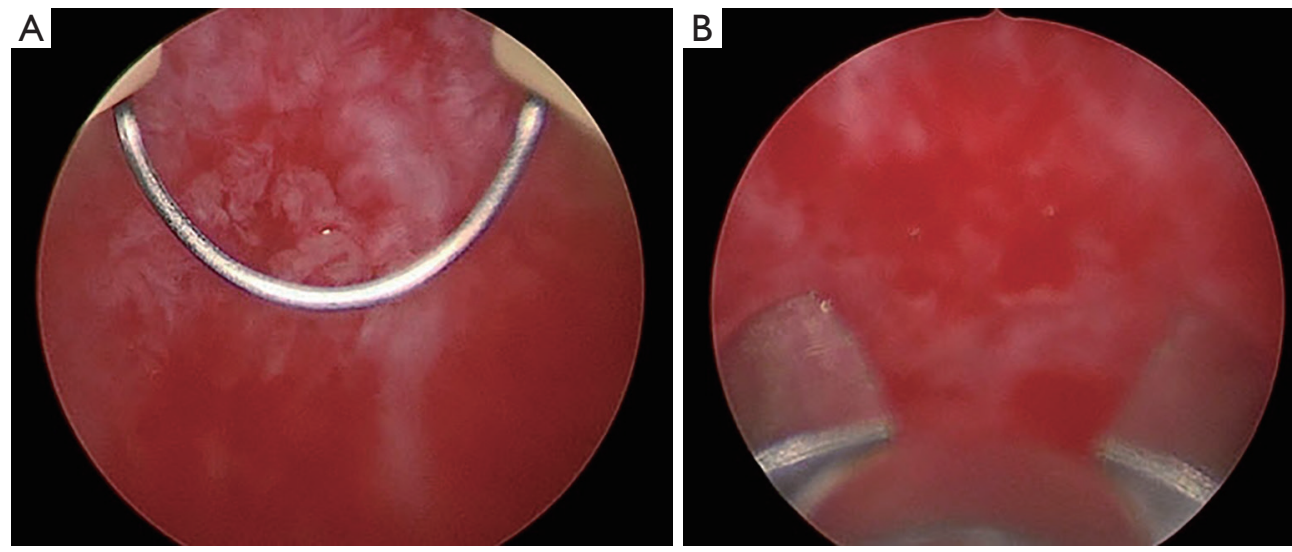

Figure 4 Bladder endoscopy with biopsy sampling.

the literature, it is decided to start with vesical instillations with dimethyl sulfoxide. We have experience using this substance for the treatment of interstitial cystitis and complex regional pain syndrome. Furthermore, it is easily available and generally well tolerated. We decide to use the same treatment schedule as for the treatment of these diseases, starting with weekly instillations of $50 \mathrm{~mL}$, keeping the substance at least 30 minutes inside the bladder, a pattern that is maintained for 6 weeks and then monthly maintenance instillations, which will be maintained for at least a year. For the instillations, a Foley is inserted just to introduce the substance and then the patient empties the bladder spontaneously.

It is worth highlighting the complete resolution of the 

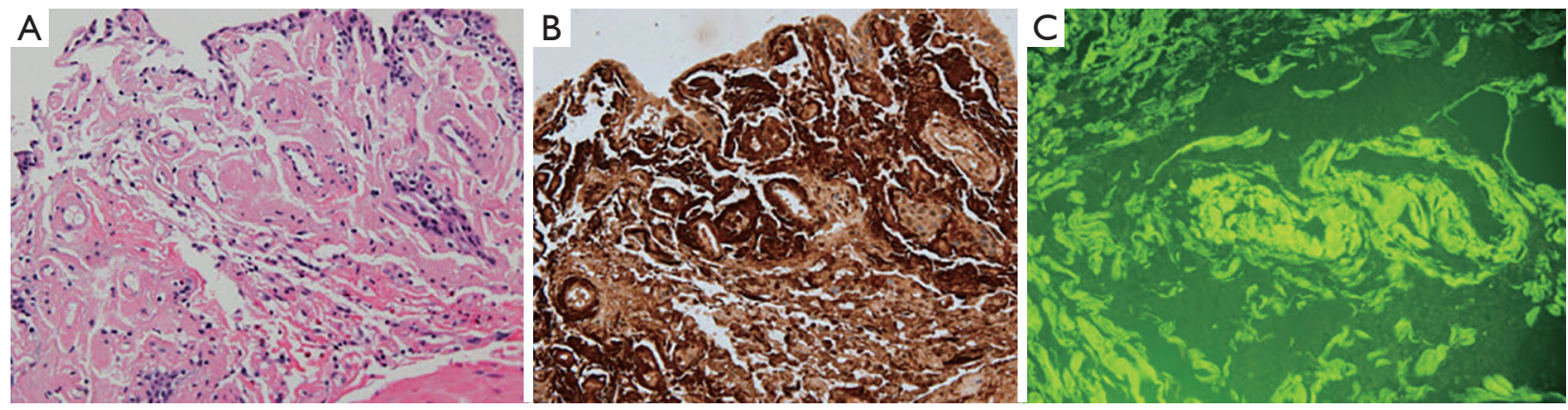

Figure 5 Bladder biopsies ( $\times 200)$. (A) Deposit of perivascular eosinophilic material in hematoxylin/eosin stain; (B) characterization of amyloid type by amyloid-specific amyloid AA immunohistochemical technique; (C) thioflavine histochemical technique, with positive perivascular eosinophilic deposits.

hematuria after the first instillation, with the possibility of the catheter removal within 2 days. The patient remains with good voiding quality without irritative symptoms and without recurrence of hematuria after 6 months of followup. The instillations were well tolerated and no related complications were observed.

Subsequently, at the 7th month of evolution, the patient developed an episode of macroscopic hematuria that required transfusion of 2 units of packed red blood cells, an endoscopic exploration with evacuation of clots and a selective embolization of the hypogastric arteries to control it. After these measures, the patient progressed favorably, remaining with clear urine and blood analysis with stable hemoglobin level. It was decided to resume the instillations with DMSO, remaining clinically stable and without hematuria for 4 months after the last episode. The total follow-up is 11 months.

\section{Discussion}

Amyloidosis is a condition that includes multiple manifestations derived from the abnormal deposition of amyloid protein in tissues. Secondary amyloidosis is reactive to chronic inflammatory processes (2).

In the urinary tract, kidney involvement occurs frequently in secondary amyloidosis (3). In our case, we do not have a renal biopsy, but there are data in favor of renal affectation, such as the existence of renal failure and proteinuria, although not with nephrotic range. In contrast, involvement of the lower urinary tract is uncommon, although its manifestations may be important (26).

The bladder is usually affected in cases of primary amyloidosis (6), being secondary amyloidosis involvement infrequent, with very few cases published in the literature $(4,11)$. Rheumatoid arthritis has been described as the disease most commonly related to these cases, unlike our case, that is secondary to chronic bronchiectasis with frequent superinfections.

The most frequent clinical manifestation of bladder amyloidosis is hematuria, with or without irritative voiding symptoms, and it is often a massive hematuria with even fatal outcomes (11). It is common for a transurethral catheter to act as a trigger for massive hematuria, as in our case $(27,28)$. Its diagnosis is sometimes a real challenge, due to the lack of symptoms specificity and unusual cystoscopic findings $(7,29)$. Its management should be sequential, from less to more aggressive treatments.

The administration of bladder instillations with dimethyl sulfoxide (DMSO) as a local treatment after endoscopic resection, seems to be an effective option for the control of this entity in recurrent cases of both primary and secondary bladder amyloidosis (6,21-23). The actual action mechanism of this substance is unknown, but it has been observed to cause a degradation of the amyloid fibers, without significant side effects $(30,31)$. Internal use, skin application and enema administration have been successfully tried since the efficacy of DMSO for amyloidosis was reported (32). This therapy is usually used orally in cases of systemic amyloidosis or gastric localized amyloidosis $(18,30,31,33)$. A topical application has also been used for cutaneous amyloidosis (34) and due to the high penetrance action of DMSO, which passes with a high concentration rapidly through the skin, some cases of localized amyloidosis of the urinary bladder and ureter, have been effectively treated with occlusive dressing therapy using DMSO in a impregnated gauze (35-37).

The work of Malek et al. exposes the experience of these 
instillations in 6 patients with primary bladder amyloidosis. They receive instillations every 2 weeks for 3 months, 6 months or a year, depending on the evolution. In two of the patients the therapy failed, requiring in one of them cystectomy (6).

Yoshino et al. (32) treated their case of residual primary bladder amyloidosis after TUR with percutaneous therapy with dimethyl sulfoxide, obtaining also satisfactory results after the treatment, with normalization of cystoscopy and magnetic resonance and without significant side effects.

The work of Diez Calzadilla et al., exposes another case of massive hematuria due to bladder amyloidosis secondary to a recurrent rheumatoid arthritis. After a TUR, selective embolization and instillations with aluminum potassium and DMSO every 72 hours were administered with good control of the hematuria (10). A similar case in a young patient, with the same basal pathology and recurrent hematuria, was treated by the group of Nurmi et al. with a pattern of biweekly instillations for a year (22).

Based on these previous experiences, we have designed a weekly instillation pattern that we maintain for 6 weeks, followed by monthly maintenance until we complete at least 1 year. We chose this pattern because it is the one we use with interstitial cystitis and because it is a midpoint between the ones described in the bibliography (a 72 hours pattern is difficult to implement and a biweekly pattern is too distant).

We have obtained excellent initial results with complete resolution of the hematuria from the first instillation, the patient stays asymptomatic and without recurrence at 6 months of follow-up. The instillations have been well tolerated and no related complications have appeared.

After this period, in the seventh month, the patient spontaneously, without previous urological manipulation, presented a recurrence of the hematuria with secondary anemia that required endoscopic evacuation of clots and a selective embolization of the hypogastric arteries to control it. Due to previous satisfactory response to DMSO, it is decided to return to the instillations, with a good subsequent evolution in the following 4 months.

\section{Conclusions}

Secondary bladder amyloidosis is a rare entity manifested mainly as a painless hematuria, sometimes difficult to control. The cystoscopic study shows images that vary from a polypoid mass to nonspecific inflammatory lesions, being the histopathological study essential. It seems that intravesical instillations with dimethyl sulfoxide could be established as a safe option with promising results.

\section{Acknowledgments}

We want to thank our colleagues from the Internal Medicine and Pathology departments, who managed the patient during the hospitalization period and processed the samples, helping us to make an accurate diagnosis.

\section{Footnote}

Conflicts of Interest: The authors have no conflicts of interest to declare.

Ethical Statement: The authors are accountable for all aspects of the work in ensuring that questions related to the accuracy or integrity of any part of the work are appropriately investigated and resolved. Written informed consent was obtained from the patient for publication of this Case report and any accompanying images.

\section{References}

1. Caballero giné JM, Borrat font $\mathrm{P}$, Martí picas L, et al. Amiloidosis vesical: presentación de dos casos. Actas Urológicas Españolas 2000;24:413-5.

2. López Marcelo F, Valdez YG, Hernández Pacheco J, et al. Amiloidosis secundaria. Características clínicas. Revista Medica Herediana 2013;10:45.

3. Jain M, Kumari N, Chhabra P, et al. Localized amyloidosis of urinary bladder: A diagnostic dilemma. Indian J Pathol Microbiol 2008;51:247.

4. Chitale S, Morsey M, Peat D, et al. Amyloidosis of Lower Genitourinary Tract: A Review. EAU-EBU Update Series 2007;5:70-6.

5. Silva H. Síndrome Nefrótico en 120 pacientes adultos del sexo masculino. Tesis Doctoral 1973. Universidad Peruana Cayetano Heredia, Lima-Perú.

6. Malek RS, Wahner-Roedler DL, Gertz MA, et al. Primary localized amyloidosis of the bladder: experience with dimethyl sulfoxide therapy. J Urol 2002;168:1018-20.

7. Altwairgi A. Primary amyloidosis of the urinary bladder presenting as painless heamaturia. Int J Health Sci (Qassim) 2011;5:181-5.

8. Tirzaman O, Wahner-Roedler DL, Malek RS, et al. Primary localized amyloidosis of the urinary bladder: a case series of 31 patients. Mayo Clin Proc 
2000;75:1264-8.

9. Raja K, Ahmed E, Mubarak M, et al. Primary Localized Amyloidosis of Urinary Bladder: A Case Report and Review of Literature. Nephrourol Mon 2013;5:994-6.

10. Diez Calzadilla NA, March Villalba JA, Canosa Fernández A, et al. Intractable hematuria secondary to systemic amyloidosis with bladder involvement. Arch Esp Urol 2014;67:637-41.

11. García-Escudero López A, Arruza Echevarría A, Leunda Saizar J, et al. Secondary amyloidosis of the bladder and massive hematuria. Actas Urol Esp 2010;34:111-5.

12. Yalcin V, Citgez S, Agaoglu I. Localised primary amyloidosis of the urinary bladder: case series of two patients. BMJ Case Rep 2009;2009. doi: 10.1136/ bcr.12.2008.1367.

13. Sato M, Kawabata S, Mizuno N, et al. Primary localized al amyloidosis of the bladder mimicking recurrence of bladder carcinoma. Nippon Hinyokika Gakkai Zasshi 2018;109:140-3.

14. Fitzpatrick R, Paterson NR, Belanger EC, et al. Primary amyloidosis of the bladder; a mimicker of bladder cancer. Can J Urol 2017;24:8868-70.

15. Patel P, Pourmandi M, Lombard JS. Primary amyloidosis of the bladder mimicking probable malignancy: A case report. Urol Case Rep 2019;26:100944.

16. Linke RP, Gärtner HV, Michels H. High-sensitivity diagnosis of AA amyloidosis using Congo red and immunohistochemistry detects missed amyloid deposits. J Histochem Cytochem 1995;43:863-9.

17. Missen GAK, Tribe CR. Catastrophic haemorrhage from the bladder due to unrecognised secondary amyloidosis. $\mathrm{Br}$ J Urol 1970;42:43-9.

18. Wang WJ, Lin CS, Wong CK. Response of systemic amyloidosis to dimethyl sulfoxide. J Am Acad Dermatol 1986;15:402-5.

19. Kito S, Itoga E, Inokawa M, et al. Studies on biological actions of dimethyl sulfoxide in familial amyloidosis. Ann N Y Acad Sci 1983;411:52-66.

20. Agarwal SK, Walmsley BH, Marley NJ. Primary amyloidosis of urinary bladder. J R Soc Med 1995;88:171P-172P.

21. McCammon KA, Lentzner AN, Moriarty RP, et al. Intravesical dimethyl sulfoxide for primary amyloidosis of the bladder. Urology 1998;52:1136-8.

22. Nurmi MJ, Ekfors TO, Rajala PO, et al. Intravesical Dimethyl Sulfoxide Instillations in the Treatment of Secondary Amyloidosis of the Bladder. J Urol 1990;143:808-10.
23. Nishiyama T, Gejyo F, Katayama Y, et al. Primary Localized Amyloidosis of the Bladder: A Case of AL (\&lambda;) Amyloid Protein and Combination Therapy Using Dimethyl Sulfoxide and Cepharanthin. Urol Int 1992;48:228-31.

24. Khan SM, Birch PJ, Bass PS, et al. Localized amyloidosis of the lower genitourinary tract: a clinicopathological and immunohistochemical study of nine cases. Histopathology 1992;21:143-7.

25. Sirohi D, Gandhi J, Amin MB, et al. Amyloidosis of the bladder and association with urothelial carcinoma: report of 29 cases. Hum Pathol 2019. [Epub ahead of print].

26. Merrimen JLO, Alkhudair WK, Gupta R. Localized amyloidosis of the urinary tract: case series of nine patients. Urology 2006;67:904-9.

27. Sanz Jaka JP, Arrinda Yeregui JM, Mendíbil Dacal J, et al. Bladder involvement in systemic amyloidosis. Arch Esp Urol 1988;41:325-8.

28. Palmero Martí JL, Budia Alba A, Arlandis Guzmán S, et al. Secondary vesical amyloidosis. Actas Urol Esp 2004;28:238-42.

29. Senarriaga Ruiz de la Illa N, Loizaga Iriarte A, Lacasa Viscasillas I, et al. Amiloidosis vesical: una lesión que simula un tumor. Actas Urol Esp 2010;34:820-1.

30. Amemori S, Iwakiri R, Endo H, et al. Oral dimethyl sulfoxide for systemic amyloid A amyloidosis complication in chronic inflammatory disease: a retrospective patient chart review. J Gastroenterol 2006;41:444-9.

31. Scheinberg MA, Pernambuco JC, Benson MD. DMSO and colchicine therapy in amyloid disease. Ann Rheum Dis 1984;43:421-3.

32. Yoshino T, Ohara S, Moriyama H. Occlusive dressing therapy using dimethyl sulfoxide in a patient presenting with primary localized amyloidosis of the urinary bladder: a case report. J Med Case Rep 2013;7:191.

33. Jin SZ, Qu B, Han MZ, et al. Endoscopic submucosal dissection combined with orally administered dimethyl sulfoxide for primary gastric localized amyloidosis. Clin Res Hepatol Gastroenterol 2014;38:e79-83.

34. Weidner T, Illing T, Elsner P. Primary Localized Cutaneous Amyloidosis: A Systematic Treatment Review. Am J Clin Dermatol 2017;18:629-42.

35. Okumura A, Morii A, Takagawa K, et al. A Case of Localized Amyloidosis of the Right Ureter Treated Effectively by Occulusive Dressing Technique Therapy Using Dimethyl Sulfoxide: Long- 
Term Follow-Up of Eight Years. Hinyokika Kiyo 2019;65:117-21.

36. Takeda T, Kozakai N, Ikeuchi K. Localized amyloidosis of the bladder treated effectively by occlusive dressing technique therapy using Dimethyl sulfoxide (DMSO): two case reports. Nippon Hinyokika Gakkai Zasshi

Cite this article as: García Rojo E, González Padilla DA, Castelo Loureiro A, Parrilla Rubio L, Tejido Sánchez Á, Guerrero Ramos F. New case of secondary bladder amyloidosis with massive hematuria-role of intravesical instillations with dimethyl sulfoxide. Transl Androl Urol 2019;8(5):548-555. doi: 10.21037/tau.2019.09.22
2005;96:705-8.

37. Kato Y, Sue Y, Fujii H, et al. Localized amyloidosis of the ureter and bladder treated effectively by occlusive dressing technique therapy using dimethyl sulfoxide: a case report. Hinyokika Kiyo 2000;46:421-4. 